\title{
Relationships among rat numbers, abundance of oil palm fruit and damage levels to fruit in an oil palm plantation.
}

\begin{abstract}
The relationships between vertebrate pests and crop damage are often complex and difficult to study. In palm oil plantations rodents remain the major pests, causing substantial monetary losses. The present study examined the numerical and functional responses of rodents to changes in the availability of oil palm fruit and the damage associated with that response. For the study, 200 traps were set in pairs on a $10 \times 10$ trapping grid for 3 consecutive nights in each of 6 study plots at 8-week intervals in a 2569 ha oil palm plantation at Labu, Negeri Sembilan state in Peninsular Malaysia over 14 months. A total of 1292 individual rats were captured over 25200 trap-nights. Animals were identified, aged, sexed, weighed and measured. An index of the relative abundance of rats was calculated based on trapping success. Damage to infructescences was assessed at each trap point. Regardless of the age of palms, there were positive and significant relationships between the relative abundance of rats and numbers of infructescences. The levels of damage to infructescences were significantly correlated with the relative abundance of rats. A steep increase in damage was observed with an increase in mature infructescences, indicating a feeding preference of rats for mature infructescences. For both males and females of all rat species, there were weak and non-significant correlations between body condition and infructescence numbers. These results indicated that there was a numerical and a functional response by rats to the availability of palm fruit and a resulting increase in depredation of oil palm fruits. The ways in which this information might aid in future pest control are discussed.
\end{abstract}

\section{Item Article \\ Type:}

Keyword: Body condition index; Fruit damage; Numerical response; Oil palms; Rodent pest. 